\title{
COST-UTILITY ANALYSIS OF BEHAVIORAL INTERVENTIONS FOR HIV-INFECTED PERSONS TO REDUCE HIV TRANSMISSION IN THE USA
}

\author{
Sada Soorapanth \\ San Francisco State University \\ Decision Sciences Department \\ 1600 Holloway Avenue \\ San Francisco, CA 94132, USA
}

\author{
Stephen E. Chick \\ INSEAD \\ Technology and Operations Management Area \\ Boulevard de Constance \\ 77300 Fontainebleau, FRANCE
}

\begin{abstract}
We developed an ordinary differential equation model to analyze the cost and utility (measured in quality adjusted life years, or QALYs) of three published behavioral HIV interventions that aim to reduce the risk of transmission from HIV-infected persons to their sexual partners. The ODE model maps measurements of behavioral risk reduction parameters, estimated from sampling, into costs and QALYs. Monte Carlo sampling was used to perform a probabilistic sensitivity analysis to quantify uncertainty in costs and QALYs due to parameter estimation error from sampling. The results suggested that the behavioral interventions considered in this study are most likely to be cost-saving, or at least cost-effective. Our study shows how statistical estimates of behavioral measures translates into uncertainty about health costs and outcomes and suggests implications for which data are important to collect when assessing cost-utility tradeoffs, and not just measures of risk reduction from behavioral interventions.
\end{abstract}

\section{INTRODUCTION}

The Centers for Disease Control (CDC) estimated that, at the end of 2006, 1.1 million people were living with HIV in the USA, and that 56,300 people were newly-infected each year. Because of the success of HIV treatment in recent years, HIV-infected persons live longer. At the same time, the challenge of managing the risk of transmission to the uninfected population remains. The CDC announced the Advancing HIV Prevention initiative to intensify HIV prevention efforts. One of the initiative's four strategies was to reduce risky behaviors among HIV-infected persons which could lead to the transmission of HIV to uninfected sexual partners (Janssen et al. 2003).

A number of studies have demonstrated the efficacy of interventions in reducing the risk behaviors. The efficacy findings from these studies, however, were measured in various forms of risk behaviors, such as the increase in condom use and the reduction in number of HIV-negative and unknown-status partners (Johnson et al. 2006, Herbst et al. 2005, Lyles et al. 2007) Without a common measure of efficacy, the benefits of these interventions cannot be evaluated against each other and compared with other types of HIV intervention prevention programs.

Cost-utility analysis (CUA) is a standard approach for comparing and evaluating multiple health interventions. CUA is based on common measures of effectiveness, such as quality-adjusted live years and costs associated with the interventions. The results from a CUA would be useful for public health policy makers in determining which intervention would be effective with best values and hence allow them to make informed decisions in allocating limited resources for HIV prevention. 


\section{Soorapanth and Chick}

Based on our literature search, there are a limited number of cost-effectiveness or CUA studies for HIV behavioral interventions. These studies did not focus on interventions for HIV-positive adults in the US and were mostly conducted before the year 2001 .

This study develops a mathematical model to assess the costs and benefits (as measured in QALYs) of three behavioral interventions for HIV-infected individuals. We consider behavioral studies of intervention programs delivered to individuals or small groups whose participants were heterosexual men and women in the USA. The outcomes of the analysis in this paper include the cost per HIV infection prevented and the cost per quality-adjusted life-year saved, although the papers that reported on the behavioral studies had a different outcome, such as the percent reduction of certain risky behaviors. Because the parameter estimates that behavioral studies are based on a statistical analysis, we also provide a probabilistic sensitivity analysis (PSA) that quantifies uncertainty about the potential costs and benefits of the interventions in both monetary and health terms. The model was implemented in Microsoft Excel spreadsheets with design goals of being transparent, user-friendly, and usable by cross-disciplinary collaborators.

\section{METHODS}

We developed a simple compartmental model of HIV transmission that is described in Section 2.1. The parameters of that modeled were varied by matching them to general population statistics as well as to parameter estimates of how different behavioral interventions change the HIV dynamics. The interventions therefore change the overall cost burden of HIV/AIDS in both monetary and health terms. We measured health benefits and costs with the model in Section 2.1. Section 2.2 describes three such behavioral interventions. The parameter estimates for the behavioral interventions are estimated with a finite set of statistics and therefore exhibit sampling error. Section 2.3 summarizes how we used the uncertainty about the estimates of risk reduction parameters to generate Monte Carlo samples from the posterior distribution of those parameters. That technique was done to enable a Bayesian probabilistic sensitivity analysis (PSA) to quantify the uncertainty about the costs and benefits of the behavioral interventions that we study, and that are due to sampling error for key infection transmission parameters. The output of the PSA was used with a cost-utility assessment as summarized in Section 2.4.

\subsection{HIV Infection Model}

We used the compartmental model in Figure 1 to describe the HIV infection transmission dynamics. The parameters of the model were varied through time in an attempt to assess how the changes in the behaviors of partners were influenced by the behavioral interventions. By running the model we could assess the change in the number of HIV transmissions from index cases to their partners. We used that information to assess the lifetime QALYs lost and lifetime treatment costs that are associated with such HIV transmissions. In this way, we assessed the incremental costs and QALYs associated with an intervention as compared without an intervention.

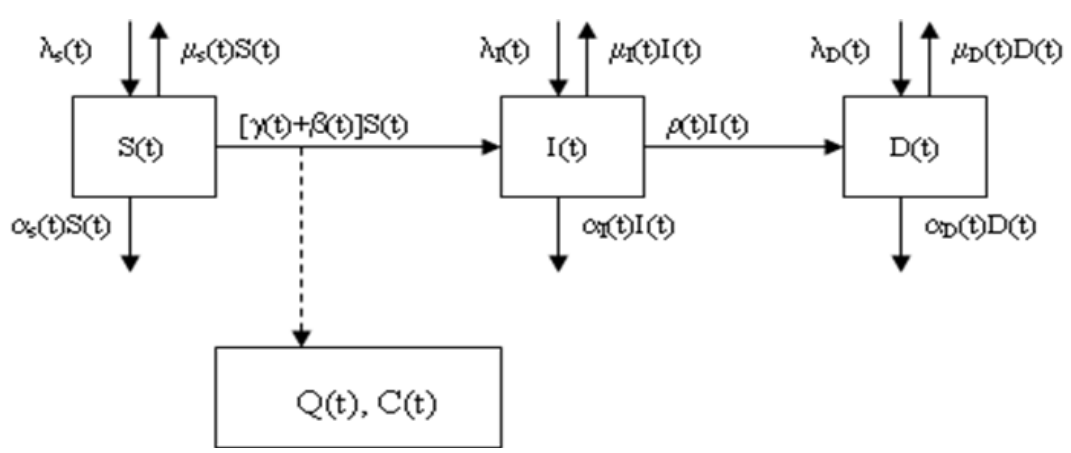

Figure 1: Continuous-Time System Dynamic Infection Model of Sexual Partners per Index Case. 


\section{Soorapanth and Chick}

In this model, an HIV-positive individual participating in the behavioral intervention program was referred to as index case. The variable $S(t)$ describes the number of susceptible partners of index cases, per index case in the behavioral intervention, as a function of time. For example, if there are 100 index cases at a given time, and there are 325 partners of those index cases that are not HIV positive, then $S(t)=3.25$ at that time. There are other state variables and a number of parameters that describe the dynamics of the model.

We describe the parameters of the compartmental model by describing the terms of its corresponding ordinary differential equation (ODE) model. That ODE is given in Equations (1) to (5).

$$
\begin{aligned}
& \frac{d S(t)}{d t}=\lambda_{s}(\mathrm{t})-\left[\mu_{s}(t)+\gamma(t)+\beta(t)+\alpha_{s}(t)\right] S(t) \\
& \frac{d I(t)}{d t}=\lambda_{\mathrm{I}}(\mathrm{t})+[\gamma(t)+\beta(t)] S(t)-\left[\mu_{I}(t)+\rho(t)+\alpha_{I}(t)\right] I(t) \\
& \frac{d D(t)}{d t}=\lambda_{\mathrm{D}}(\mathrm{t})+\rho(t) I(t)-\left[\mu_{D}(t)+\alpha_{D}(t)\right] D(t) \\
& \frac{d Q(t)}{d t}=[\gamma(t)+\beta(t)] S(t) q e^{-r t / n} \\
& \frac{d C(t)}{d t}=[\gamma(t)+\beta(t)] S(t) c e^{-r t / n}
\end{aligned}
$$

Equation (1) describes the rate of change in the number of susceptible partners per index case, $S(t)$, over time. The term $\lambda_{S}(t)$ in the compartmental model determines the rate that an index case acquires new susceptible sexual partners. The value of that rate was set by dividing the mean number of HIV-negative sexual partners per index case by the mean duration of a partnership. The term $\mu_{S}(t)$ is the rate that a susceptible partner leaves the partnership with the index case per time period. The rate that a susceptible partner becomes infected during the partnership with an index case is $\gamma(t)+\beta(t)$, where $\gamma(t)$ is the rate of infection transmission from the index case and $\beta(t)$ is the transmission rate due to sexual contacts with other HIV-infected individuals in the general population (other than the index case). The death rate of partners that are susceptible to HIV is $\alpha_{S}(t)$.

Equations (2) and (3) describe the rates of change in the number of asymptotically infected partners per index case, $I(t)$, and the rate of change in the number of those with AIDS per index case, $D(t)$. The first terms of those equations, $\lambda_{I}(t)$ and $\lambda_{D}(t)$, are the acquiring rate of asymptomatically infected sexual partners and that of partners with AIDS, respectively. As was $\lambda_{S}(t), \lambda_{I}(t)$ and $\lambda_{D}(t)$ were estimated by dividing the mean numbers of asymptomatically infected partners and the mean numbers of partners with AIDS, by the duration of partnership. The rates of leaving a partnership with an index case are $\mu_{I}(t)$ and $\mu_{D}(t)$ for an asymptomatically infected partner and for a partner with AIDS, respectively. Both $\mu_{I}(t)$ and $\mu_{D}(t)$ were assumed to be equal to $\mu_{S}(t)$. An infected individual develops AIDS conditions at the rate of $\rho(t)$. The death rate of partners with AIDS conditions is $\alpha_{D}(t)$. It is assumed that all infected individuals developed AIDS conditions before they died, hence, $\alpha_{I}(t)$, was assumed to be zero.

Each newly infected partner incurs a lifetime HIV treatment costs of $c$, and a loss of $q$ qualityadjusted life years (QALYs). The total lifetime HIV treatment costs, $C(t)$ and the total QALYs lost, $Q(t)$, associated with newly infected partners increases at the rate described by Equations (4) and (5). The rates were calculated by multiplying the rate of newly infected among all partners per index case, $[\gamma(t)+$ $\beta(t)] S(t)$, by lifetime treatment costs per infections (c) in Equation (4) and by the QALYs lost per infection $(q)$ in Equation (5). Both rates were discounted at the annual discount rate of $r \%$. We divided the discount rate $r$ by $n$, the number of time periods per year, to obtain the discount rate per time period.

Now that the model is formalized, we turn to the specification of the parameters of the model. The transmission rate, $\gamma(t)$, is defined by noting that the number of protected sexual contacts (i.e. with condom 
use) causing infection per time period, $x_{p}$, is a Poisson random variable with the mean of $m_{p}(t) p_{p}$, and the number of unprotected sexual contacts (i.e. sexual contact without condom use), $x_{u}$, is a Poisson random variable with the mean of $m_{u}(t) p_{u}$. The means $m_{p}(t)$ and $m_{u}(t)$ are the mean number of protected and unprotected sexual contacts per individual. The probabilities $p_{p}$ and $p_{u}$ are the transmission probabilities per protected contact and per unprotected contact respectively. Let $P(t)$ be the probability that at least one infection occurs during the time period $t$ to $t+d t$. Given this non-homogeneous Poisson process model for infection arrivals and our adaptation of the model for the finite difference (FD) tools below, we have:

$$
\begin{gathered}
P(t)=P\left(x_{p}>0\right)+P\left(x_{u}>0\right)-P\left(x_{p}>0\right) P\left(x_{u}>0\right) \\
\quad=\left(1-\exp \left(-m_{p}(t) p_{p} d t\right)\left(1-\exp \left(-m_{u}(t) p_{u} d t\right) .\right.\right.
\end{gathered}
$$

The rate of infection at time $t, \gamma(t)$, therefore, are modeled by $-[\ln (1-P(t))] / d t$.

The transmission rate $\beta(t)$ between susceptible partners and other HIV-infected individual in the general population (excluding the index case) is modeled by

$$
\beta(t)=\frac{k}{y}(y-1) P_{H I V} p_{u}
$$

where $k$ is the number of unprotected sexual contacts per period among heterosexual women and men in the US, $y$ is the number of sexual partners per individual among heterosexual women and men in the US, and $P_{H I V}$ is the HIV prevalence among US population. The term $k / y$ approximates the number of unprotected sexual contacts per sexual partner per period. The number of HIV-positive sexual partners, excluding the index case equals $(y-1) * P_{H I V}$. By multiplying that with the transmission probability per unprotected sexual contact, $p_{u}$, we obtain the rate of transmission $\beta(t)$. In the last term of Equation (1), the death rate of susceptible partners, $\alpha_{S}(t)$, was estimated by the annual death rate among US adults.

We simulated this ODE model in a spreadsheet by using the Euler-forward method (Brennan, Chick, and Davies 2006). This method, while offering less numerical stability than some other methods of computing with ODE models, offers an ease of implementation in spreadsheets. This method updates states on a discrete time grid, such as by

$$
x_{j+1}=x_{j}+f\left(x_{j}, t_{j}\right) \Delta t
$$

where $\mathrm{x}_{\mathrm{j}}$ is the state of the system at time $t_{j}=j \Delta t$ and $f\left(x_{j}, t_{j}\right)=d x / d t$. In this study, we used a 3-month time period (i.e. $\Delta t=3$ months), an interval that is consistent with the time interval reported in most behavioral studies. We followed the dynamic of the system over the period of 20 years.

All costs in the model were converted to 2009 US\$ using the consumer price index for medical care (US Bureau of Labor Statistics 2009). The model's parameter values are summarized in Tables 1 and 2.

\subsection{HIV Behavioral Intervention for HIV-Positive Individuals}

We searched the published literature on HIV behavioral interventions, including meta-analytic and systematic reviews (Lyles et al. 2007, Johnson et al. 2005, Herbst et al. 2005, Kelly and Kalichman 2002, Crepaz, Lyles, and Wolitski 2006). We focused on individual and small group interventions for HIV-infected individuals that aimed at changing behaviors through counseling. We only considered studies with quantitative results, and therefore did not study those with dichotomous or categorical measures (such as Wolitski et al. 2005, Richardson et al. 2004). 
Table 1: Parameter Values for the HIV Transmission System

\begin{tabular}{|c|c|c|}
\hline Parameter & Base-Case & Reference \\
\hline HIV prevalence among US population & 0.0100 & Sanders et al. 2005 \\
\hline Transition rate from HIV to AIDS (events per patient-year) & 0.0585 & Sanders et al. 2005 \\
\hline Transition rate from HIV to death (events per patient-year) & 0.0000 & Assumption \\
\hline Transition rate from AIDS to death (events per patient-year) & 0.0339 & Sanders et al. 2005 \\
\hline Average annual, age-adjusted death rate in US (2003-2005) & 0.0081 & $\begin{array}{l}\text { National Center for } \\
\text { Health Statistics } 2009\end{array}$ \\
\hline Percent reduction in transmission probability from condom use & $90 \%$ & $\begin{array}{l}\text { Cohen, } \mathrm{Wu} \text {, and Far- } \\
\text { ley } 2004\end{array}$ \\
\hline $\begin{array}{l}\text { Per-act transmission probability (unprotected, male-to-female } \\
\text { vaginal sex) }\end{array}$ & 0.001 & $\begin{array}{c}\text { Cohen, Wu, and Far- } \\
\text { ley } 2004\end{array}$ \\
\hline Average duration of partnership (years) & 1 & Assumption \\
\hline $\begin{array}{l}\text { Number of unprotected vaginal/anal sex with HIV-negative } \\
\text { partners per 3-month among heterosexual women and men in } \\
\text { the US }\end{array}$ & 11.3 & Weinhardt et al. 2004 \\
\hline $\begin{array}{l}\text { Number of unprotected vaginal/anal sex with HIV-positive } \\
\text { partners per 3-month among heterosexual women and men in } \\
\text { the US }\end{array}$ & 13.55 & Weinhardt et al. 2004 \\
\hline $\begin{array}{l}\text { Number of sexual partners over past 3-months among hetero- } \\
\text { sexual women and men in the US }\end{array}$ & 1.7 & Weinhardt et al. 2004 \\
\hline Number of QALYs lost per HIV infection & 8.22 & Pinkerton et al. 2000 \\
\hline $\begin{array}{l}\text { Lifetime cost of treating HIV/AIDS costs per infection (in } \\
2009 \text { US } \$ \text { ) }\end{array}$ & $\$ 466,579$ & Shackman et al. 2006 \\
\hline
\end{tabular}

Three studies were included in our analysis (Table 2). Although some interventions also targeted drug risk behaviors, we only focused on sexual risk behaviors in our analysis. Kalichman et al. (2001) examined group intervention focused on strategies for practicing safer sex. Patterson et al. (2003) studied a brief counseling that focused on condom use, safer-sex negotiation, and serostatus disclosure. The intervention of Rotheram-Borus et al. (2004) consisted of two-module counseling focused on coping with one's serostatus and staying healthy, and reducing substance use and unprotected sexual acts.

Table 2: Parameter Values for Risky Behaviors for Each Behavioral Intervention

\begin{tabular}{|c|c|l|}
\hline Study & Setting & Parameter Values: Without intervention; With Intervention \\
\hline $\begin{array}{c}\text { Kalichman } \\
\text { et al. (2001) }\end{array}$ & $\begin{array}{c}\text { Community- } \\
\text { based service } \\
\text { agency }\end{array}$ & $\begin{array}{l}\text { Mean (SE) number of sex partners: 1.6 (0.18); 1.2 (0.14) } \\
\text { Mean number of unprotected sex acts (vaginal and anal) in the past 3 } \\
\text { months: 2.7 (0.67); 1.2 (0.33) }\end{array}$ \\
\hline $\begin{array}{c}\text { Patterson et } \\
\text { al. (2003) }\end{array}$ & $\begin{array}{c}\text { Clinic settings } \\
\text { where HIV+ } \\
\text { seek cares }\end{array}$ & $\begin{array}{l}\text { Mean number of HIV-negative or unknown-serostatus sexual partners } \\
\text { over the past 4 months: 7.9 (0.83); 7.9 (0.83) } \\
\text { Number of unprotected sex acts (vaginal and anal) with HIV-negative } \\
\text { or unknown serostatus sexual partners over the past 4 months: 34.7 } \\
\text { (2.68); 20.2 (1.88) }\end{array}$ \\
\hline $\begin{array}{c}\text { Rotheram- } \\
\text { Borus et al. } \\
\text { (2001) }\end{array}$ & $\begin{array}{c}\text { Clinical care } \\
\text { sites }\end{array}$ & $\begin{array}{l}\text { Mean number of HIV-negative sexual partners: 4 (0.57); 1.4 (0.2) } \\
\text { Percentage of unprotected sex acts (vaginal and anal) with HIV- } \\
\text { negative partners: 47\%; 27\% } \\
\text { Mean number of unprotected sex acts (vaginal and anal) with HIV- } \\
\text { negative partners per 3-month (estimated by combining with data } \\
\text { from Weinhardt et al. 2004): 11.3 (3.19); 6.49 (1.83) }\end{array}$ \\
\hline \multicolumn{2}{|l}{}
\end{tabular}




\section{Soorapanth and Chick}

Patterson et al. (2003) did not report the mean number of sexual partners after the intervention was delivered. We therefore used the same mean values in both the "with" and the "without" intervention scenarios. Rotheram-Borus et al. (2001) did not report the mean number of unprotected sex acts but rather a percentage value. We estimated the mean number of unprotected acts by multiplying the percentage value by the mean number of sex acts per 3-month period among US adults from Weinhardt at al. (2004), studying almost 4,000 HIV-positive individuals in four major US cities. The standard errors of the mean values were computed from the standard deviations of the outcomes reported in the intervention studies where possible. Rotheram-Borus et al. did not report such standard deviations. We therefore estimated the standard errors of this intervention using similar data from Weinhardt et al. (2004).

Only one of the studies (Rotheram-Borus et al. 2004) reported the costs of intervention (also called program costs). For the other two studies, we estimated the program costs based on provided details about intervention delivery, including the number of program staff delivering the intervention, the total number of sessions, the session duration, and the average number of clients served per session. We assumed that the program's delivery staff - or facilitators - would have an education background similar to that of a medical and public health social worker, and multiplied the total program delivery time by the average hourly wage of a social worker in the United States in 2008 (\$22.87, US Bureau of Labor Statistics, 2008) plus $30 \%$ for fringe benefits (total, $\$ 29.73$ per hour). We divided by the number of clients to get the perclient facilitator costs.

In order to estimate more complete program costs, including those for recruitment, training, supervision, administration, supplies, equipment, facility space and participant costs, we examined two additional cost studies involving HIV-infected person. One was an ART adherence case management program in Los Angeles County persons in which clients met one-on-one with a case manager for 14 times on average during a six-month period to address barriers to ART adherence (Garland et al. 2007). The other was a 10-session program for up to 15 participants per session to reduce risk behaviors among HIV-infected injection drug users in four U.S. cities (Tuli et al. 2005).

Based on the data from these two costs studies, the proportion of the cost of a case manager's time to the total societal costs was estimated at 0.25 . Therefore, we assumed that the facilitator costs estimated for the program considered in this study were $25 \%$ of societal costs. To arrive at societal costs, we divided the facilitator costs by $25 \%$. Table 3 summarizes the estimated counselor times and expenses required by each intervention. The program cost of the intervention in Rotheram-Borus et al. (2004) was reported at $\$ 3500$ per participant (in 2002 US\$). The cost was adjusted to 2009 US\$ using the consumer price index of medical care (US Bureau of Labor Statistics, 2009), resulting in the program cost of $\$ 4603.10$ per participant.

Table 3: Summary of Program Cost Calculations for Kalichman et al. (2001) and Patterson et al. (2003):

\begin{tabular}{|c|l|c|c|c|}
\hline Intervention & Intervention's details & $\begin{array}{c}\text { Total Counse- } \\
\text { Tor's Cost per } \\
\text { Time*Hourly Pay } \\
\text { Participant }\end{array}$ & $\begin{array}{c}\text { Total Program } \\
\text { Cost per } \\
\text { Participant }\end{array}$ \\
\hline $\begin{array}{c}\text { Kalichman } \\
\text { et al. 2001 }\end{array}$ & $\begin{array}{l}\text { Number of session:5, } \\
\text { Length of session: 2 hours, } \\
\text { Number of counselors: 2, } \\
\text { Number of participants: } 8 .\end{array}$ & US\$ 594.6 & US\$ 74.33 & US\$ 297.3 \\
\hline $\begin{array}{c}\text { Patterson et } \\
\text { al. 2003 }\end{array}$ & $\begin{array}{l}\text { Number of session:3, } \\
\text { Length of session: 1.5 hours, } \\
\text { Number of counselors: 2, } \\
\text { Number of participants: } 8 .\end{array}$ & US\$267.57 & US\$ 33.45 & US\$ 133.8 \\
\hline
\end{tabular}




\section{Soorapanth and Chick}

\subsection{Probabilistic Sensitivity Analysis}

We used Monte Carlo sampling to explore the effect of parameter uncertainty on the cost and effectiveness results. Since we focused on the effect of intervention on the risky sexual behaviors and the infection transmissions among partners, we only sampled parameters related to sexual behaviors. Table 4 presented the selected model parameters and the sampling distributions. We assumed that the number of sexual partners and the number of unprotected sexual contacts follow gamma distributions. The parameter of gamma distribution, $\alpha$ and $\beta$ were derived from the mean $(\mu=\alpha \beta)$ and standard errors $\left(S E=s q r t\left(\alpha \beta^{2}\right)\right)$, i.e. $\alpha=\mu^{2} / S E^{2}$, and $\beta=S E^{2} / \mu$.

Table 4: Monte Carlo Sampling for Probabilistic Sensitivity Analysis

\begin{tabular}{|c|c|c|}
\hline Intervention & Model Parameter & Sampling Distribution \\
\hline $\begin{array}{l}\text { Kalichman } \\
\text { et al. (2001) }\end{array}$ & $\begin{array}{l}\text { Mean number of sexual partners in the past } 3 \text { months: } \\
\text { Without Intervention; } \\
\text { With intervention } \\
\text { Mean number of unprotected sex acts (vaginal and anal) in the } \\
\text { past } 3 \text { months: } \\
\text { Without Intervention; } \\
\text { With intervention }\end{array}$ & $\begin{array}{l}\text { Gamma }(82.22,0.019) \text {; } \\
\text { Gamma }(73.80,0.016) \\
\text { Gamma }(16.29,0.166) \text {; } \\
\text { Gamma }(13.16,0.091)\end{array}$ \\
\hline $\begin{array}{l}\text { Patterson et } \\
\text { al. (2003) }\end{array}$ & $\begin{array}{l}\text { Mean number of HIV-negative or unknow-serostatus sexual } \\
\text { partners over the past } 4 \text { months: } \\
\text { Without Intervention; } \\
\text { With intervention } \\
\text { Mean number of unprotected sex acts (vaginal and anal) with } \\
\text { HIV-negative or unknown serostatus sexual partners over the } \\
\text { past } 4 \text { months: } \\
\text { Without Intervention; } \\
\text { With intervention }\end{array}$ & $\begin{array}{l}\text { Gamma }(89.80,0.088) \\
\text { Gamma }(89.80,0.088) \\
\text { Gamma }(167.78, \\
0.207) ; \\
\text { Gamma }(115.35,0.175)\end{array}$ \\
\hline $\begin{array}{l}\text { Rotheram- } \\
\text { Borus et al. } \\
(2001)\end{array}$ & $\begin{array}{l}\text { Mean number of HIV-negative sexual partners: } \\
\text { Without Intervention; } \\
\text { With intervention } \\
\text { Mean number of unprotected sex acts (vaginal and anal) with } \\
\text { HIV-negative partners per 3-month: } \\
\text { Without Intervention; } \\
\text { With intervention }\end{array}$ & $\begin{array}{l}\text { Gamma }(49.00,0.082) \\
\text { Gamma }(47.06,0.03)\end{array}$ \\
\hline
\end{tabular}

\subsection{Cost-Utility Analysis}

For each intervention, the model was run for 1000 iterations using parameters from Tables 2-4. In each simulation run, the model calculated the total number of infections among partners per index case, the discounted QALYs lost per index case, and the discounted HIV lifetime treatment costs per index case, in both With-intervention and Without-Intervention scenarios. The method of common random numbers was used for the two scenarios.

The mean discounted lifetime HIV treatment costs saved by the intervention is the mean of the differences in the discounted lifetime HIV treatment costs in the 'With Intervention' and 'Without Intervention' scenarios. Similarly, the mean discounted QALYs saved by the intervention is the mean of the differences in the discounted QALYs lost in the two scenarios. The incremental cost-effectiveness ratio (ICER) is

$$
I C E R=\frac{\text { Program Costs }- \text { Mean Discounted Lifetime HIV treatment costs saved }}{\text { Mean Discounted QALYs saved }}
$$




\section{Soorapanth and Chick}

The program is considered cost-saving if the saving of total lifetime HIV treatment cost exceeds the program costs. In which case, the ICER will be negative. The program is considered cost-effective if the ICER is less than or equal to $\$ 50,000$, a widely used threshold in public health economic evaluation.

\section{RESULTS}

Table 5-7 shows the cost and effectiveness analyses of the three interventions as compared to the "Without Intervention" scenario. These values were based upon our simulated estimates of the mean. When the index case received a risk reduction intervention and modified their risk behaviors for 1 year, the mean (SE) discounted number of infections prevented were $0.2254(0.0035), 1.41(0.01)$, and $0.75(0.02)$ per index case for the interventions in Kalichman et al., Patterson et al., and Rotheram-Borus et al., respectively. The prevention of these HIV infections resulted in the mean (SE) discounted QALY saved of 1.85 (0.03), $11.56(0.09)$, and $6.16(0.14)$ per index case, respectively. On average, the intervention would save the discounted lifetime treatment cost of HIV by $\$ 105,175(\mathrm{SE}=1,634), \$ 656,257(\mathrm{SE}=5,106)$, and $\$ 349,926$ ( $\mathrm{SE}=8,050$ ) per index case, for Kalichman et al., Patterson et al., and Rotheram-Borus et al., respectively. The distributions of the lifetime HIV treatment cost saving are shown in Figure 2

All interventions were cost-saving when the model used the sample mean as a point estimator of the given parameters. When uncertainty was accounted for in the parameter estimates, we estimate that the probability that an intervention was cost-saving or at least cost-effective was $98.5 \%, 99.9 \%$ and $92 \%$ for Kalichman et al., Patterson et al. and Rotheram-Borus et al., respectively, based on 1000 Monte Carlo samples from the posterior distribution of the behavioral risk parameters.

\section{CONCLUSIONS}

We conducted a cost-effectiveness analysis of behavioral risk reduction interventions which aim to reduce risk behaviors of HIV-positive individuals. This was needed in order to extend the variety of intermediate outcomes reported elsewhere into cost-effectiveness values that can be used to compare across various interventions. To model the range of intervention's efficacies, the outcomes from three HIV intervention studies were considered in the analysis. The costs of intervention, if not available, were estimated based on the details of the intervention delivery published in the studies.

The results suggested that behavioral interventions, such as those in Kalichman et al.(2001), Patterson et al (2003) and Rotheram-Borus et al. (2001), can potentially reduce the risk of HIV transmission, particularly if the behavioral changes are sustained over time. Information regarding the duration of intervention effects and the duration of sexual partnership is important to better estimate transmission risk and intervention effectiveness. The duration of these behavioral changes seem to strongly influence the magnitude of the cost effectiveness of these programs, but the duration of effectiveness seems to be an open question. The cost-effectiveness analyses also showed that the three interventions studied above were most likely to be cost-saving, or at least cost-effective, because the program cost estimates were much lower than the savings of lifetime treatment cost of HIV. Among the three interventions, Patterson et al. appeared to cost least and save more QALYs than the other interventions.

The robustness of the cost and effectiveness results depends significantly on the assumptions made to overcome three main challenges encountered in this study. First was the lack of comparable sexual behaviors data from the three studies to be able to project the number of infections averted and QALY saved. Secondly, not all studies provided a measure of variation of these behavioral outcomes. In order to obtain such data, we used the baseline behavior data from Weinhardt et al. (2004), conducted from relative large sample size of HIV-positive individuals in the US. Thirdly, the intervention program costs were not available in the publications. We estimated these costs based on available information and assumption about the resource use. The costs could vary significantly if the interventions were to be applied in practice. With these limitations, our study should be considered as an analysis of potential impacts of a behavioral intervention on the risk behaviors, estimated in terms of cost and final health outcomes. 
Table 5: Mean and Standard Errors (SE) of Costs and Health Outcomes When Comparing the Without and With Intervention From Kalichman et al. (2001).

\begin{tabular}{lccc}
\hline Outcome & $\begin{array}{c}\text { Without } \\
\text { Intervention }\end{array}$ & $\begin{array}{c}\text { With } \\
\text { Intervention }\end{array}$ & Difference \\
\hline Mean (SE) number of partners' infections per in- & 1.29 & 1.07 & 0.2254 \\
dex case & $(0.01)$ & $(0.01)$ & $(0.0035)$ \\
Mean(SE) discounted QALYs lost due to part- & 10.63 & 8.77 & 1.85 \\
ners' infections per index case & $(0.08)$ & $(0.06)$ & $(0.03)$ \\
Mean (SE) discounted lifetime treatment costs & $\$ 603,233$ & $\$ 498,058$ & $\$ 105,175$ \\
due to partners' infections per index case & $(\$ 4,673)$ & $(\$ 3,300)$ & $(\$ 1,634)$ \\
Program cost per index case & $\$ 0$ & $\$ 297.30$ & $\$ 297.30$ \\
\hline Incremental costs per QALY saved (ICER) & - & $-\$ 56,601$ & \\
Proportion of Runs with negative ICER (cost- & & 0.985 & \\
saving) or with ICER $\leq \$ 50,000$ (cost-effective) & & & \\
\hline
\end{tabular}

Table 6: Mean and Standard Errors (SE) of Costs and Health Outcomes When Comparing the Without and With Intervention From Patterson et al. (2003).

\begin{tabular}{lccc}
\hline Outcome & $\begin{array}{c}\text { Without } \\
\text { Intervention }\end{array}$ & $\begin{array}{c}\text { With } \\
\text { Intervention }\end{array}$ & Difference \\
\hline Mean (SE) number of partners' infections per in- & 19.59 & 18.19 & 1.41 \\
dex case & $(0.06)$ & $(0.05)$ & $(0.01)$ \\
Mean(SE) discounted QALYs lost due to part- & 161.05 & 149.49 & 11.56 \\
ners' infections per index case & $(0.46)$ & $(0.40)$ & $(0.09)$ \\
Mean (SE) discounted lifetime treatment costs & $\$ 9,141,468$ & $\$ 8,485,211$ & $\$ 656,257$ \\
due to partners' infections per index case & $(\$ 26,166)$ & $(\$ 22,706)$ & $(\$ 5,106)$ \\
Program cost per index case & $\$ 0$ & $\$ 133.8$ & $\$ 133.8$ \\
\hline Incremental costs per QALY saved & & $-\$ 56,750$ & \\
Proportion of Runs with negative ICER (cost- & & 0.999 & \\
saving) or with ICER $\leq \$ 50,000$ (cost-effective) & & & \\
\hline
\end{tabular}

Table 7: Mean and Standard Errors (SE) of Costs and Health Outcomes When Comparing the Without and With Intervention From Rotheram-Borus et al. (2001).

\begin{tabular}{lccc}
\hline Outcome & $\begin{array}{c}\text { Without } \\
\text { Intervention }\end{array}$ & $\begin{array}{c}\text { With } \\
\text { Intervention }\end{array}$ & Difference \\
\hline Mean (SE) number of partners' infections per in- & 6.62 & 5.87 & 0.75 \\
dex case & $(0.06)$ & $(0.04)$ & $(0.02)$ \\
Mean(SE) discounted QALYs lost due to part- & 54.44 & 48.27 & 6.16 \\
ners' infections per index case & $(0.47)$ & $(0.36)$ & $(0.14)$ \\
Mean (SE) discounted lifetime treatment costs & $\$ 3,089,964$ & $\$ 2,740,038$ & $\$ 349,926$ \\
due to partners' infections per index case & $(\$ 26,896)$ & $(\$ 20,356)$ & $(\$ 8,050)$ \\
Program cost per index case & $\$ 0$ & $\$ 4603.10$ & $\$ 4603.10$ \\
\hline Incremental costs per QALY saved & - & $-\$ 56,015$ & \\
Proportion of Runs with negative ICER (cost- & & 0.92 & \\
saving) or with ICER $\leq \$ 50,000$ (cost-effective) & & & \\
\hline
\end{tabular}



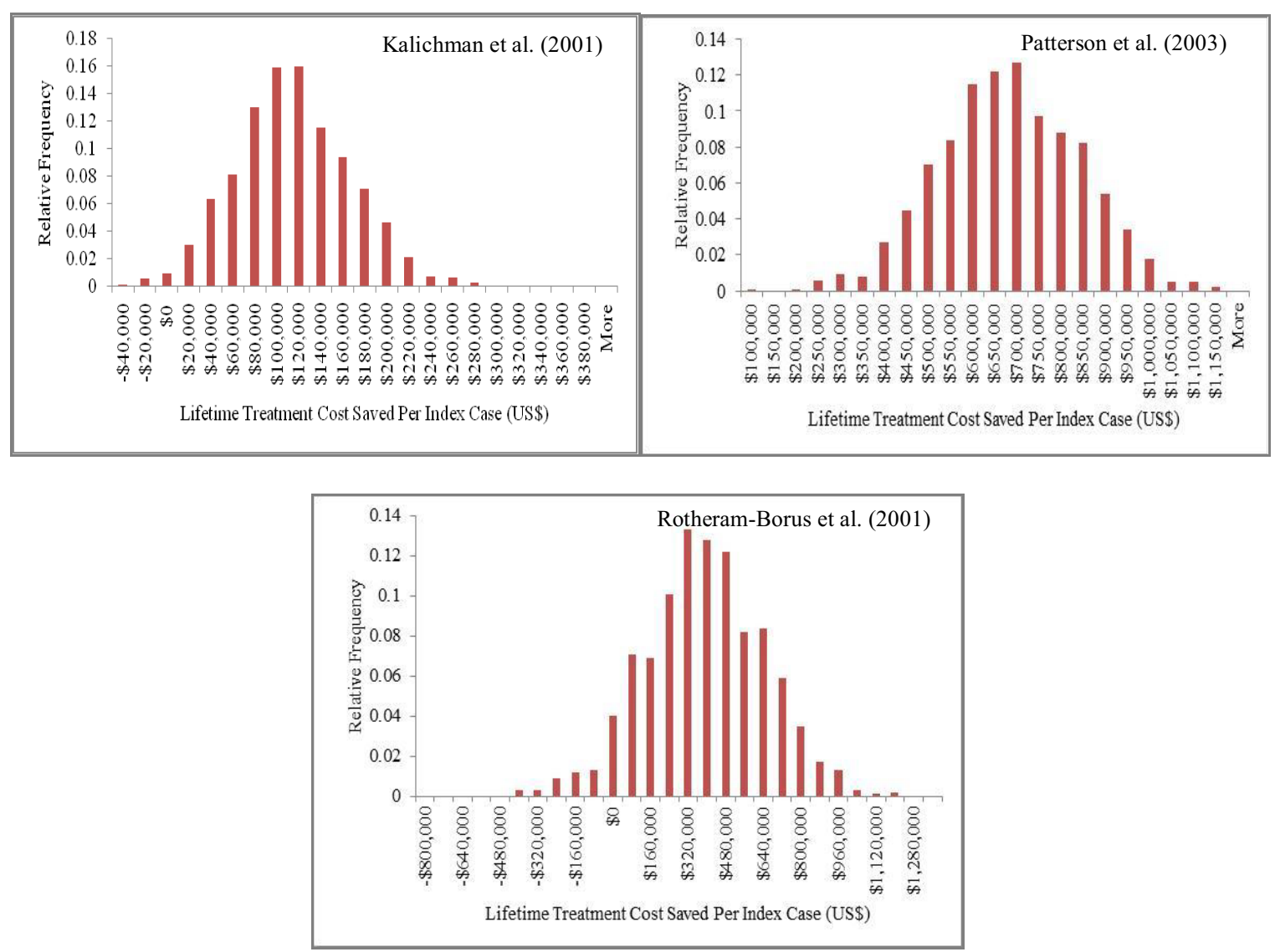

Figure 2: Relative Frequency Distribution of Life Time Treatment Costs Saved Per Index Case (Based on 1000 Monte Carlo's Simulation Runs)

\section{REFERENCES}

Brennan, A., S. Chick, and R. Davies. 2006. A taxonomy of model structures for economic evaluation of health technologies. Health Economics 15:1295-1310.

Cohen, D., S.Y. Wu, and T. Farley. 2004. Comparing the cost-effectiveness of HIV prevention interventions. Journal of Acquired Immune Deficiency Syndrome 37:1404-1414.

Crepaz, N., C. Lyles, and R. Wolitski. 2006. Do prevention interventions reduce HIV risk behaviours among people living with HIV? A meta-analytic review of controlled trials. AIDS 20:143-157.

Garland, W., A. Wohl, R. Valencia et al. 2007. The acceptability of a directly-administered antiretroviral therapy (DAART) intervention among patients in public HIV clinics in Los Angeles, California. AIDS Care 19:159-167.

Herbst, J., R. Sherba, N. Crepaz et al. 2005. A meta-analytic review of HIV behavioral interventions for reducing sexual risk behavior of men who have sex with men. Journal of Acquired Immune Deficiency Syndrome 39: 228-241.

Janssen, R., I. Onorato, R. Valdiserri et al. 2003. Advancing HIV Prevention: New strategies for a changing epidemic --- United States. Morbidity and Mortality Weekly Report 52: 329-332.

Johnson, B., M. Carey, S. Chaudoir et al. 2006. Journal of Acquired Immune Deficiency Syndrome 41: 642-650.

Kalichman, S., D. Rompa, M. Cage et al. 2001. Effectiveness of an intervention to reduce HIV transmission risks in HIV-positive people. American Journal of Preventive Medicine 21: 84-92. 
Kelly, J., and S. Kalichman. 2002. Behavioral research in HIV/AIDS primary and secondary prevention: recent advances and future directions. Journal of Consulting and Clinical Psychology 70:626-639.

Lyles, C., L. Kay, N. Crepaz, et al. 2007. Best-evidence interventions: findings from a systematic review of HIV behavioral interventions for US populations at high risk, 2000-2004. American Journal of Public Health 97: 133-143.

National Center for Health Statistics. Health, United States, 2008, With Cartbook, Hyattville, MD: 2009. Available at: <http://www.cdc.gov/nchs/data/hus/hus08.pdf\#026> [accessed September 16, 2009]

Patterson, T., W. Shaw, and S. Semple. 2003. Reducing the sexual risk behaviors of HIV+ Individuals: outcome of a randomized controlled trial. Annals of Behavioral Medicine 25: 137-145.

Pinkerton, S., D. Holtgrave, W. DiFranceisco et al. 2000. Cost-threshold analyses of the National AIDS demonstration research HIV prevention interventions. AIDS 14: 1257-1268.

Richardson, J., J. Milam, A. McCutchan. 2004. Effect of brief safer-sex counseling by medical providers to HIV-1 seropositive patients: a multi-clinic assessment. AIDS 18: 1179-1186.

Rotheram-Borus, M., M. Lee, D. Murphy et al. 2001. Efficacy of a preventive intervention for youths living with HIV. American Journal of Public Health 9: 400-405.

Sanders, G., A. Bayoumi, V. Sundaram et al. 2005. Cost-effectiveness of screening for HIV in the era of highly active antiretroviral therapy. New England Journal of Medicine 352: 570-85.

Schackman, B., K. Gebo, R. Walensky et al. 2006. The lifetime cost of current human immunodeficiency virus care in the United States. Medical Care 44: 990-997.

Tuli, K., S. Sansom, D. Purcell et al. 2005. Economic evaluation of an HIV prevention intervention for seropositive injection drug users. Journal of Public Health Management and Practice 11: 508-515.

US Bureau of Labor Statistics. 2009. Consumer price index databases. Available at: <http://www.bls.gov/cpi/cpi_dr.htm> [accessed April 6, 2010].

US Bureau of Labor Statistics. 2008. Occupational Employment and Wages, May 2008. Available at: <http://www.bls.gov/oes/2008/may/oes211022.htm\#nat> [accessed April 6, 2010].

Weinhardt, L., J. Kelly, M. Brondino et al. 2004. HIV transmission risk behavior among men and women living with HIV in 4 cities in the United States. Journal of Acquired Immune Deficiency Syndrome 36: 1057-66.

Wolitsky, R., C. Gomez, J. Parson et al. 2005. Effects of a peer-led behavioral intervention to reduce HIV transmission and promote serostatus disclosure among HIV-seropositive gay and bisexual men. AIDS 19: S99-S109.

\section{AUTHOR BIOGRAPHIES}

SADA SOORAPANTH is an assistant professor at the department of decision sciences, San Francisco State University (SFSU). Prior to joining SFSU, she was a Prevention Effectiveness Fellow assigned to the Division of HIV/AIDS Prevention, Centers for Disease Control and Prevention in Atlanta, GA. Her main research interests are in epidemic modeling and economic evaluation of health policy and medical technology. She has also worked with major pharmaceutical companies to perform economic modeling and analyses in various diagnostic and therapeutic areas. Her email address is <sada@sfsu.edu>.

STEPHEN E. CHICK is a professor of Technology and Operations Management at INSEAD, and the Novartis Chair of Healthcare Management. He has worked in the automotive and software sectors prior to joining academia, and now teaches operations with applications in manufacturing and services, particularly the health care sector. He enjoys Bayesian statistics, stochastic models, and simulation. His web page is <faculty. insead.edu/chick/> and email address is <stephen. chicklinsead.edu>. 